In der Rubrik „Literatur kompakt" werden die wichtigsten Originalarbeiten aus der internationalen Fachliteratur referiert.

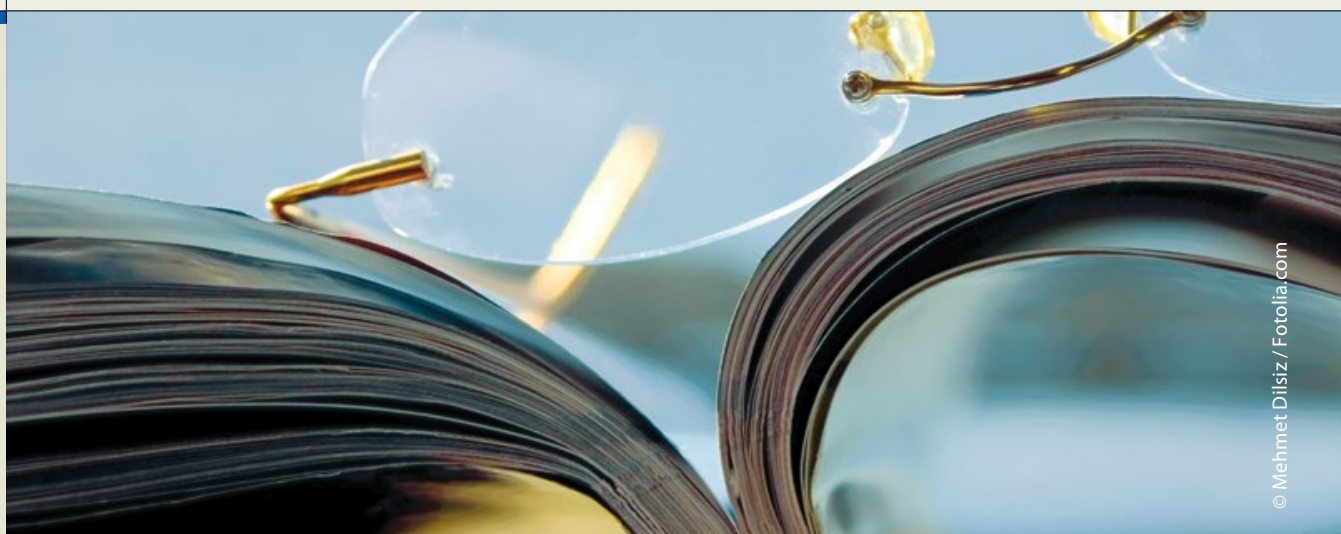

\section{Melanomrisiko durch Potenzmittel?}

\section{Männer, die PDE-5-Hemmer einnehmen, entwickeln etwas häufiger Melanome. Möglicherweise liegt das aber nicht an den Medikamenten, sondern am Lebensstil von Potenzmittel-Anwendern.}

D ie Sorge, Hemmer der Phosphodiesterase-5 (PDE-5) könnten möglicherweise die Entstehung maligner Melanome fördern, gründet ursprünglich auf dem Wirkmechanismus der Medikamente. Ihr Zielenzym ist an einem wichtigen Signalweg der Melanomentwicklung beteiligt: Bei Mutationen im BRAFGen wird die PDE-5 herunterreguliert, was einen Anstieg des Kalziums im Zytoplasma und letztlich eine höhere Invasivität von Melanomzellen zur Folge hat. Zudem wurde in der Health-Professionals-Follow-up-Studie ein vermehrtes Auftreten von Melanomen bei SildenafilAnwendern beschrieben. Ein internationales Ärzteteam hat den Zusammenhang deswegen anhand von schwedischen Registerdaten erneut überprüft.
Die Fall-Kontroll-Studie zeigt ebenfalls eine signifikant erhöhte Rate von Melanomen bei Anwendern von PDE-5-Hemmern. Von 4.065 Männern, bei denen zwischen 2006 und 2012 ein Melanom festgestellt wurde, hatten 435 (11\%) zuvor PDE-5-Hemmer eingenommen. In der Kontrollgruppe mit 20.325 Männern ohne Hautkrebs-Diagnose waren es nur 1.713 (8\%) gewesen. Die Nutzer von PDE5-Hemmern hatten damit, unabhängig von anderen Risikofaktoren, eine signifikant höhere Melanomrate (Odds Ratio, OR 1,21; 95\%-KI 1,08-1,36).

Eine Dosis-Wirkungs-Beziehung war jedoch nicht festzustellen. Das Melanomrisiko war nur dann signifikant erhöht, wenn die Männer nicht mehr als ein Rezept für PDE-5-Inhibitoren erhalten hat-

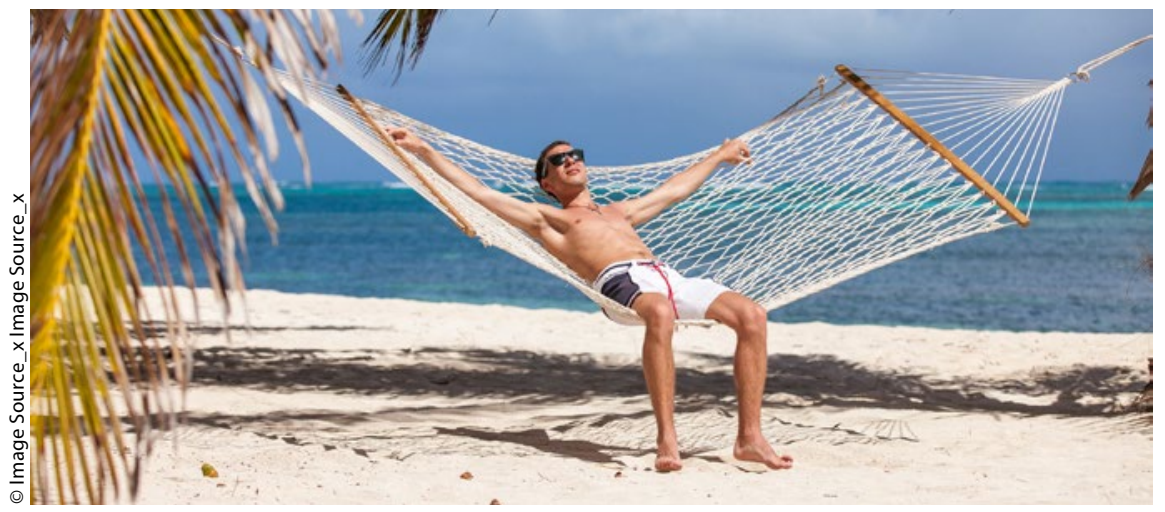

Wer sich viel der Sonne aussetzt, hat ein erhöhtes Melanomrisiko - unabhängig davon, ob er PDE-5-Hemmer einnimmt oder nicht. ten (OR 1,32; $95 \%$-KI 1,08-1,36; $4 \%$ vs. $3 \%)$. Die lang wirksamen PDE-5-Hemmer Tadalafil und Vardenafil waren zudem mit demselben Risiko belastet wie das kurz wirksame Sildenafil. Mit einer kausalen Beziehung kaum vereinbar waren auch die Tumorstadien: Die Anwendung der PDE-5-Hemmer korrelierte zwar mit dem Auftreten von Melanomen in den Stadien 0 und I, aber nicht mit dem Auftreten fortgeschrittener Melanome (Stadien II-IV). Auch war die Melanomrate nur erhöht, wenn mit der Einnahme von PDE-5-Hemmern erst innerhalb des letzten Jahres begonnen worden war. Als weiteres Argument gegen einen kausalen Zusammenhang gilt das Auftreten von Basalzellkarzinomen: Ihre Rate war bei den Anwendern von PDE-5-Hemmern ebenfalls erhöht, obwohl der beschriebene Signalweg über PDE-5 dabei keine Rolle spielt.

Fazit: Männer, die PDE-5-Inhibitoren anwenden, haben eine erhöhte Melanomrate. Das Assoziationsmuster spricht jedoch eher gegen einen kausalen Zusammenhang: Die Verbindung könnte demnach den Einfluss von Lebensstilfaktoren widerspiegeln, die sowohl mit dem Gebrauch von PDE-5-Hemmern als auch mit dem Auftreten niedriggradiger Melanome verbunden sind: So verfügten beide Personengruppen über einen höheren Bildungsgrad und ein höheres Einkommen. Daher würden sie möglicherweise mehr private Reisen unternehmen und seien daher stärker sonnenbrandgefährdet.

Dr. Beate Schumacher 